\title{
Refractory subretinal fluid in patients with neovascular age-related macular degeneration treated with intravitreal ranibizumab: visual acuity outcome
}

\author{
Liuna Jang • Christina Gianniou • Aude Ambresin • \\ Irmela Mantel
}

Received: 31 January 2014 /Revised: 29 July 2014 / Accepted: 27 August 2014 / Published online: 30 September 2014

(C) Springer-Verlag Berlin Heidelberg 2014

\begin{abstract}
Purpose To investigate the functional outcome of eyes with neovascular AMD (nAMD) and subretinal fluid (SRF) refractory to treatment with ranibizumab.

Methods Retrospective chart review of consecutive treatment-refractory SRF in nAMD despite monthly ranibizumab injections during 12 months or more. Data were evaluated for baseline characteristics, location of the refractory SRF, mean visual acuity (VA) change, number of injections, and timepoint of first complete disappearance of SRF.

Results Forty-five eyes in 44 patients (mean age of 76 years) were included. The mean follow-up was 32.4 months (range 12-73 months). The mean number of injections was 11.6 in the first year and 27.5 over follow-up. The refractory SRF was located subfoveally in $66.7 \%$. In 12 eyes $(26.7 \%)$, complete absorption of SRF was found after a mean of 22.6 months (range, 13-41 months). Mean VA increased by 10.4, 8.2, and 8.6 letters by month 12,24 , and 36 , respectively.

Conclusions Neovascular AMD with SRF refractory to monthly retreatment with ranibizumab may still allow good and maintained visual improvement, even if the fluid is located subfoveally. SRF may progressively absorb under continuous monthly treatment. The necessity to treat refractory SRF
\end{abstract}

\footnotetext{
L. Jang $\cdot$ C. Gianniou $\cdot$ A. Ambresin $\cdot$ I. Mantel

Department of Ophthalmology, University of Lausanne, Lausanne, Switzerland

L. Jang $\cdot$ C. Gianniou $\cdot$ A. Ambresin $\cdot$ I. Mantel

Jules Gonin Eye Hospital, Lausanne, Switzerland

L. Jang $\cdot$ C. Gianniou $\cdot$ A. Ambresin $\cdot$ I. Mantel

Fondation Asile des aveugles, Lausanne, Switzerland

I. Mantel $(\bowtie)$

University Eye Hospital Jules Gonin, 15 Av. de France - Case postale

133, 1000 Lausanne, Switzerland

e-mail: i.mantel.widmer@gmx.ch
}

with monthly injections could be questioned and would need future investigations.

Keywords Neovascular age-related macular degeneration . Refractory subretinal fluid · Ranibizumab · Anti-VEGF . Intravitreal injections

\section{Introduction}

Currently, the gold standard treatment for neovascular AMD (nAMD) is intravitreal anti-VEGF. Ranibizumab was the first anti-VEGF molecule that showed average improvement of visual acuity (VA) on the basis of monthly intravitreal reinjections [1, 2]. Subsequent studies have attempted to obtain the same favorable functional results while applying a reduced number of intravitreal injections [3-10]. In most of these studies, optical coherence tomography (OCT) has become an essential investigation method in order to determine the exudative activity of nAMD and therefore the indication for retreatment. However, individual need for retreatment varies widely, and some patients require monthly injections even in pro re nata regimen due to persistent signs of exudative activity on OCT [3-5].

The natural course of nAMD is associated with progressive BCVA loss due to the exudative activity and extension of the choroidal neovascularization (CNV), and the subsequent fibrotic and/or atrophic scarring process. Persistent or frequently recurrent exudative activity in cases of ranibizumab-treated nAMD may therefore lead to reduced functional benefit of the treatment over time. This has been well shown in studies with reduced fixed 3-monthly regimen [7, 8]. It has also been shown that monthly ranibizumab injections may not prevent BCVA loss in all cases [1,2], and that such functional loss is associated with CNV extension [11]. Therefore, persistent exudative activity on OCT despite maximal monthly 
treatment might be associated with poor functional prognosis, and the utility of treatment may be questioned.

The aim of this study was to examine the functional outcome of nAMD patients with subretinal fluid (SRF) refractory to monthly re-injections with ranibizumab in order to describe the prognosis and estimate the usefulness of treatment in these cases.

\section{Methods}

This retrospective study was performed in the medical retina service at the Jules-Gonin University Eye Hospital at Lausanne, Switzerland. The study was approved by the Swiss Federal Department of Health for retrospective analysis of the data, and was performed in accordance with the ethical standards laid down in the Declaration of Helsinki.

All AMD charts were reviewed between March 2006 (ranibizumab commercially available for treatment for nAMD) until June 2013, in order to identify the consecutive series of patients with nAMD with the following inclusion criteria: nAMD, treatment naïve at baseline, followed by treatment with intravitreal ranibizumab injections only, persistent subretinal fluid on OCT at each visit over the first 12 months of ranibizumab treatment for nAMD (age> 50 years), resulting in monthly treatment being indicated for at least 12 months from baseline. Due to the imperfect timing in real life, the number of injections was allowed to range from 10 to 13 during the first 12 months, under the condition of fluid being present at each visit 1 month after the last injection. We excluded patients with polypoidal choroidal vasculopathy, any previous macular treatment, any confounding retinal pathology, with insufficient image quality, and those with treatment refractory intraretinal fluid seen as cysts on OCT.

Patients with nAMD underwent a treatment protocol which started with 3-monthly loading doses of intravitreal ranibizumab $(0.5 \mathrm{mg})$, followed by a pro re nata $(\mathrm{PRN})$ regimen, based on monthly visits. Retreatment criteria were signs of any intra-or subretinal fluid on OCT, new hemorrhage on fundus examination, or exudation on fluorescein angiography.

Data were collected for the patient's identity, the eye, the angiographic lesion type, the follow-up duration, the location of the persistent SRF on OCT (foveal or extrafoveal), the time to successful disappearance of SRF on OCT, best-corrected visual acuity (BCVA) over time, central retinal thickness (CRT) over time, and total number of injections.

The main outcome criteria were mean visual acuity change over time, the proportion of eyes with $>15$ letters gain, $>0$ letter gain, and $<15$ letters loss. Secondary outcome criteria were the success rate of complete absorption of SRF and number of injections during follow-up. Retinal thickness measurements on OCT were not taken into account because of the use of different OCT machines.

Baseline examination and all subsequent follow-up visits included BCVA on Early Treatment of Diabetic Retinopathy (ETDRS) charts at a distance of $4 \mathrm{~m}$, a slit-lamp examination, measurement of the intraocular pressure (IOP), a dilated fundus exam, and an OCT examination (six radial lines on Stratus OCT3, Carl Zeiss Meditec, Inc., Oberkochen, Germany, until December 2008; $128 \times 512$ cube examination on SD-OCT Cirrus, Carl Zeiss Meditec, Inc., Oberkochen, Germany, from January 2009). Fundus autofluorescence (FAF) imaging, fluorescein angiography (FA), and indocyanine green angiography (ICGA) were performed at baseline using the Topcon TRC50IX (Tokyo, Japan). FAF and FA were repeated at month 3 and annually, and in addition if needed to the investigators discretion (including ICGA particularly in suboptimal treatment response).

The treatment with intravitreal ranibizumab was performed by a retina specialist according to the usual technique, each injection being $0.5 \mathrm{mg}$, performed in an operating room according to local requirements.

For data analysis, a spreadsheet on Microsoft Excel 2010 and the statistical program JMP, version 8.0.1 (SAS institute Inc., Cary NC, USA) were used. A statistical significance level of 0.05 was required.

\section{Results}

A total of 45 eyes of 44 patients were identified with treatment refractory subretinal fluid (SRF), which persisted for 12 months or longer despite monthly retreatment with ranibizumab (mean 11.6 injections in the first year, range, 10-12).

The gender was female in $27(60 \%)$ and male in 18 patients (40\%). The mean age was 76.0 years (SD 8.1). Predominantly classic lesions were found in nine eyes $(20 \%)$, minimal classic in six eyes (13\%), occult only choroidal neovascularization (CNV) in 25 eyes ( $56 \%$ ), and RAP lesions in five eyes (11\%).

The mean follow-up duration was 32.4 months (SD 15.5). The mean number of injections was 27.5 over the entire follow-up duration (SD 11.1). Most eyes (33=73.3\%) never showed complete absorption of subretinal fluid on any OCT during the entire follow-up despite continuous monthly retreatment. In 12 eyes (26.7 \%), absorption of SRF was found after a mean of 22.6 months, with a wide range from 13 to 41 months. Because of varying follow-up durations, a Kaplan-Meier success curve was calculated using completely dry retina as the success criterion. The result showed a gradual increase of this success criteria, reaching $25 \%$ success rate after 25 months and still raising afterwards $(n=23$ eyes; 
Fig. 1 Kaplan-Meier success curve of completely dry retina in initially ranibizumab-treatment refractory subretinal fluid in neovascular age-related macular degeneration

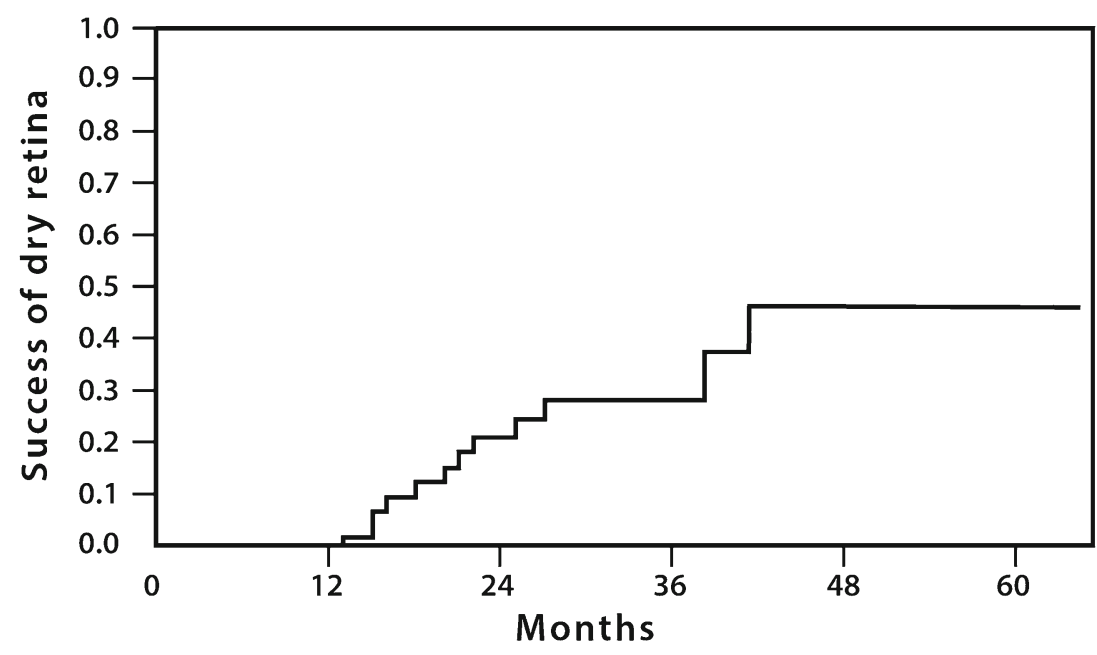

Fig. 1). The location of the refractory SRF was subfoveal in 30 eyes $(66.7 \%)$ and extrafoveal in 15 eyes $(33.3 \%)$.

Mean BCVA was 65.3 letters ETDRS (SD 11.9) at baseline. Mean BCVA increase was 7.5, 10.4, 8.2, and 8.6 letters by months $3,12,24$, and 36, respectively. Figure 2 shows the mean change of BCVA over time.

An improvement by 15 ETDRS letters or more was found in 29,36 , and $21 \%$ of eyes by months 12,24 , and 36 , respectively (data available for $n=45,33,14$ eyes at month 12,24 , and 36 , respectively, each serving as $100 \%$ ); improved BCVA by 0 letters or more was found in 84,79 , and $79 \%$ of eyes; and 98, 97, and $100 \%$ of eyes had lost less than 15 letters ETDRS. In an attempt to take into account the varying follow-up duration and exploring a potential dropout bias in our cohort, a survival curve was calculated for VA loss less than 15 letters ETDRS: at 12 and 24 months, $98 \%$ of eyes had "survived", and at 36 months $94 \%$ of eyes still had no more than VA loss.

The subgroup analysis of patients with foveal location of the refractory SRF showed no worse functional outcome than those with extrafoveal location (Fig. 3, no significant difference at any time point, $p \geq 0.15$ ). We also compared the subgroups with or without absorption during follow-up. The result graphically suggests a better outcome for eyes with absorption, however this result was statistically not significant at any time point ( $p \geq 0.066$, Fig. 4 )

Exploration of various factors potentially influencing the functional outcome (occult versus other types of CNV, presence or absence of pigment epithelium detachment at baseline, presence or absence of fibrosis or atrophy) did not show any significant association with the functional results at month 12 or month 24 ( $p>0.05, t$ test and Mann-Whitney test).

The proportion of eyes with signs of fibrosis (evaluated on fundus color photographs and FA) at baseline, 12 and 24 months was 2, 8, and $20 \%$, respectively. Signs of atrophy (evaluated on FAF) were found in 4,14 , and $16 \%$, respectively.

\section{Discussion}

The large multicenter randomized trials MARINA and ANCHOR $[1,2]$ set the gold standard for what we consider successful visual improvement in nAMD, based on monthly intravitreal injections of ranibizumab. Alternative treatment regimens have been explored [3-5, 12-14], and their justification always compares to the visual acuity improvement in
Fig. 2 Mean best-corrected visual acuity change of patients with refractory subretinal fluid despite monthly retreatment with ranibizumab, Error bars represent standard error of visual acuity change

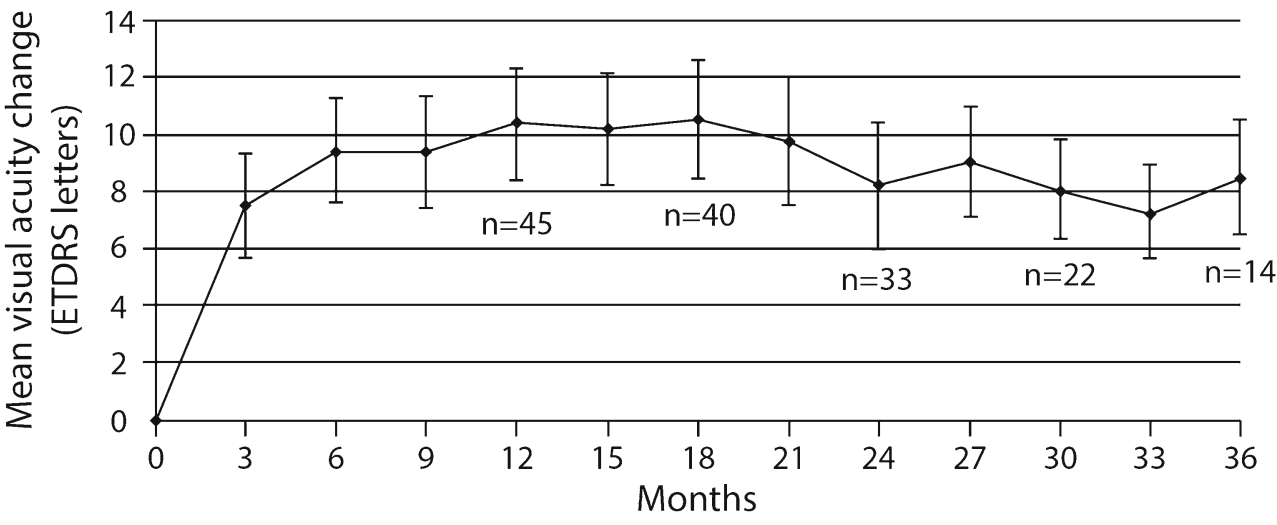


Fig. 3 Mean best-corrected visual acuity change of patients with subretinal fluid in subfoveal versus extrafoveal foveal location and refractory despite monthly retreatment with ranibizumab. Curves of mean visual acuity change are shown for follow-up of at least six eyes

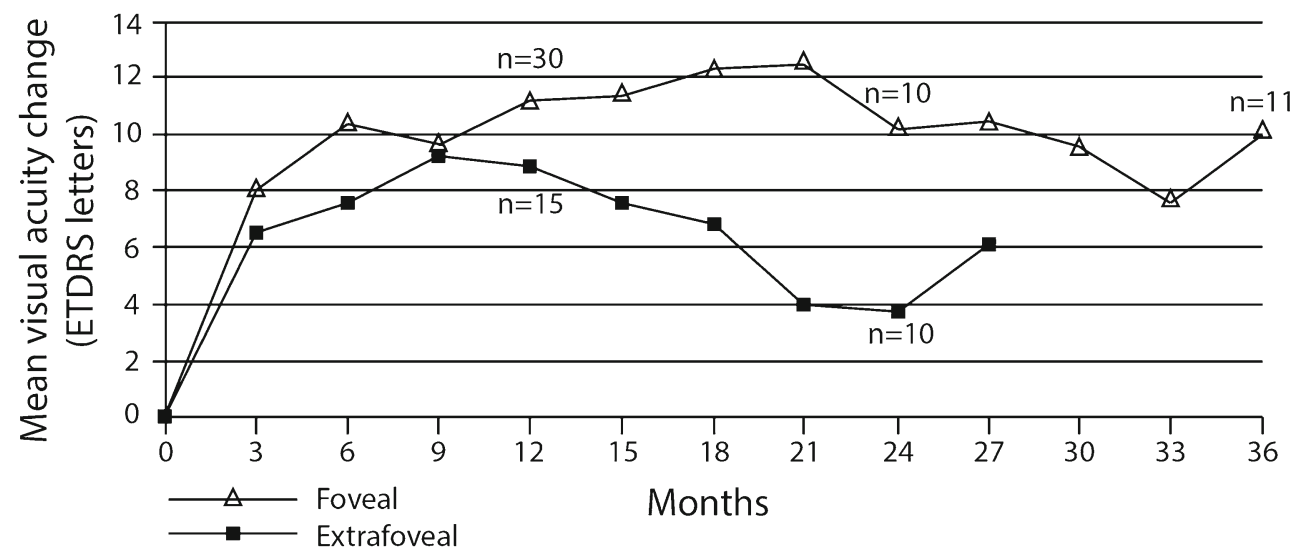

the monthly retreatment regimen. However, regimens that were associated with secondary visual acuity loss after the initial loading phase of 3-monthly injections usually apply a mean number of injections that is relatively low, whether as a fixed retreatment every 3 months [7, 8], or in a PRN with insensitive retreatment criteria [15] or in the real-life setting with less than monthly visits $[16,17]$. It has been reported that poorer outcome is typically found if the mean number of reinjections drops below five in a year $[10,18]$. The reason for this functional loss is believed to be related to multiple exudative recurrences and insufficient VEGF suppression, leading to repetitive periods of ongoing tissue damage and finally irreversible functional loss [19].

However, exudative activity, which may be seen on OCT as subretinal fluid or intraretinal cysts, may not only occur in insufficient treatment regimen, but also occur despite maximal monthly retreatment with ranibizumab. Such fluid may therefore in the same way lead to functional loss, particularly if persistent over time. The present pilot study explored this question by an analysis of the subgroup of eyes with persistent subretinal fluid (without intraretinal cysts). The results showed that refractory SRF in nAMD despite monthly retreatment with ranibizumab may still allow good function outcome. Although caution is mandatory in comparisons across studies, it is interesting that both the absolute visual acuity changes and the maintenance after month 3 in our cohort are similar to the results in the MARINA and ANCHOR trials, despite SRF at each visit for 12 months and more. It was surprising that our series of refractory SRF showed no secondary loss of the initial VA gain as compared to month 3 , even until month 36 . However, there was a trend of mild VA loss after month 18 , and this might compare to the mild, mostly insignificant secondary VA loss often found in variable dosing regimen such as PRN or treat and extend [3-6, 13].

Although we do not know whether the refractory SRF is in fact permanent or early recurrences after each of the monthly injections (mean 11.6 in the first year), we might have
Fig. 4 Mean best-corrected visual acuity change of patients with or without complete absorption of the initially refractory fluid despite monthly retreatment with ranibizumab

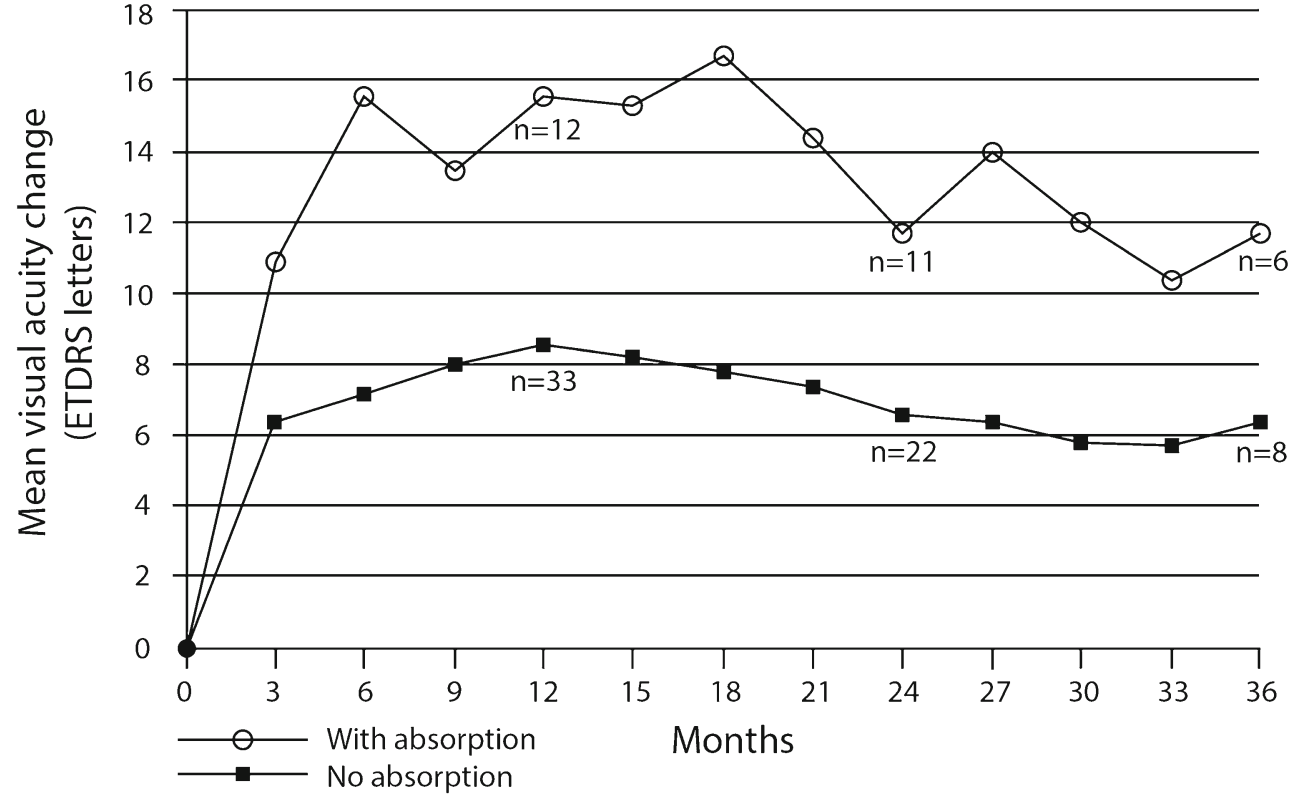


expected a stronger influence in terms of smaller visual benefit and/or secondary function loss as compared to the abovementioned studies: SRF on each monthly visit was expected to have more impact than the mean recurrence interval of 2 months in the HARBOR trial [6]. However, according to our results, the persistence of SRF did not threaten VA during at least the first 3 years, even with two-thirds being located subfoveally.

There are several possible explanations for our results: First, SRF might be an exudative component, which in itself does not greatly threaten VA. Similarly, a subgroup analysis of the ANCHOR trial found that SRF was not a risk factor for visual loss: they suggest that visual loss despite monthly injections is primarily due to the extension of $\mathrm{CNV}$, the anomaly in the RPE, and the atrophic scar [11]. Also, in central serous chorioretinopathy, although a different pathology with different characteristics of SRF, the latter does not necessarily threaten VA [20, 21]. Second, refractory SRF might differ from recurrent exudative activity. The maximal anti-VEGF therapy might induce a different molecular composition of the fluid and be of less harm than recurrent fluid. Therefore we might question the necessity of continuous monthly treatment for refractory SRF. Third, we considered the possibility of a dropout bias in our cohort, with the worst cases potentially being lost to follow-up. However, such failure would have been captured in the survival curve (more than 15 letters loss as a failure criterion) because of its independence from variable follow-up duration. The results showed no trend to support this hypothesis of a dropout bias. The incidence of this failure was even lower than reported in the large multicenter trials with monthly retreatment $[1,2]$. We therefore considered such bias absent and we exclude this third explanation.

For clinicians facing the problem of refractory fluid in nAMD under treatment with anti-VEGF, the situation may not be reassuring. As a matter of fact, in a clinical setting, we still recommend full evaluation with multimodal retinal imaging. This will allow for recognizing differential retinopathies that require a different treatment approach, such as polypoidal choroidal vascular lesions, central serous choroidopathy, or inflammatory disorders. On the other hand, it has been repeatedly suggested that switching the anti-VEGF drug might be beneficial in cases of refractory fluid. However, the benefit appears to be mainly on the structural side, and most studies found no functional improvement [22-25].

We acknowledge that the present study has several limitations. Besides the inherent weaknesses of a retrospective analysis, we need to acknowledge the variable follow-up duration, the imperfect timing of the monthly indicated reinjections (leading to slightly longer intervals), the absence of a comparison group, and the absence of meaningful data for central retinal thickness over time (varying OCT machines used). In addition, this analysis was limited to cases with refractory SRF, without intraretinal fluid. Finally, there is no information available between injections and the visits 1 month later, which does not allow the differentiation between chronic SRF and early exudative recurrence. However, even if we assume early exudative recurrences, more frequent than monthly injections might not have been indicated: first because of the good functional outcome in our cohort, and second because of the risk of accelerated development of geographic atrophy with more injections. The latter has been suggested in the results of the CATT trial, where monthly ranibizumab treatment showed less fluid on OCT and less fluorescein dye leakage, but more eyes with geographic atrophic [4]. The neuroprotective role of VEGF, as well as a potential nutritional support of the neovascularization for the retina might even support a treatment strategy that does not aim at a strictly dry retina.

In conclusion, subretinal fluid refractory to monthly treatment with ranibizumab still allows good and maintained visual improvement over 3 years, even in subfoveal location. The initially refractory SRF may disappear late (up to 41 months) under continuous monthly treatment. However, given the very good functional results, with this type of SRF well tolerated by the retina, and given the recent hypothesis of monthly injections leading to more atrophy [4], we consider that monthly retreatment might not be mandatory for refractory SRF. We might speculate that these cases could be managed with reinjections only if worsening is found as compared to the least amount of refractory SRF in the first 12 months. However, the present study does not give an answer to this question. Ideally, this question should be addressed in a prospective randomized study.

Acknowledgments This study was approved by the Swiss Federal Department of Health for retrospective analysis of the data (authorization \# 035.0003-48) and was performed in accordance with the ethical standards laid down in the Declaration of Helsinki.

Conflict of interest The authors declare that they have no conflicts of interest.

\section{References}

1. Boyer DS, Antoszyk AN, Awh CC, Bhisitkul RB, Shapiro H, Acharya NR, MARINA Study Group (2007) Subgroup analysis of the MARINA study of ranibizumab in neovascular age-related macular degeneration. Ophthalmol 114:246-252

2. Kaiser PK, Brown DM, Zhang K, Hudson HL, Holz FG, Shapiro H, Schneider S, Acharya NR (2007) Ranibizumab for predominantly classic neovascular age-related macular degeneration: subgroup analysis of first-year ANCHOR results. Am J Ophthalmol 144:850-857

3. Lalwani GA, Rosenfeld PJ, Fung AE, Dubovy SR, Michels S, Feuer W, Davis JL, Flynn HW Jr, Esquiabro M (2009) A variable-dosing regimen with intravitreal ranibizumab for neovascular age-related macular degeneration: year 2 of the PrONTO Study. Am J Ophthalmol 148:43-58 
4. Martin DF, Maguire MG, Fine SL, Ying G, Jaffe GJ, Grunwald JE, Toth C, Redford M, Ferris FL (2012) Ranibizumab and bevacizumab for treatment of neovascular age-related macular degeneration: twoyear results. Ophthalmol 119:1388-1398

5. Chakravarthy U, Harding SP, Rogers CA, Downes SM, Lotery AJ, Culliford LA, Reeves BC, IVAN study investigators (2013) Alternative treatments to inhibit VEGF in age-related choroidal neovascularisation: 2-year findings of the IVAN randomised controlled trial. Lancet 382:1258-1267

6. Busbee BG, Ho AC, Brown DM, Heier JS, Suñer IJ, Li Z, Rubio RG, Pf L (2013) Twelve-month efficacy and safety of $0.5 \mathrm{mg}$ or $2.0 \mathrm{mg}$ ranibizumab in patients with subfoveal neovascular age-related macular degeneration. Ophthalmol 120:1046-1056

7. Schmidt-Erfurth U, Eldem B, Guymer R, Korobelnik JF, Schlingemann RO, Axer-Siegel R, Wiedemann P, Simader C, Gekkieva M, Weichselberger A, EXCITE Study Group (2011) Efficacy and safety of monthly versus quarterly ranibizumab treatment in neovascular age-related macular degeneration: the EXCITE study. Ophthalmol 118:831-839

8. Abraham P, Yue H, Wilson L (2010) Randomized, double-masked, sham-controlled trial of ranibizumab for neovascular age-related macular degeneration: PIER study year 2. Am J Ophthalmol 150: 315-324

9. Holz FG, Amoaku W, Donate J, Guymer RH, Kellner U, Schlingemann RO, Weichselberger A, Staurenghi G, SUSTAIN Study Group (2011) Safety and efficacy of a flexible dosing regimen of ranibizumab in neovascular age-related macular degeneration: the SUSTAIN study. Ophthalmol 118:663-671

10. Dadgostar H, Ventura AACM, Chung JY, Sharma S, Kaiser PK (2009) Evaluation of injection frequency and visual acuity outcomes for ranibizumab monotherapy in exudative age-related macular degeneration. Ophthalmol 116:1740-1747

11. Rosenfeld Philip J, Shapiro H, Tuomi L, Webster M, Elledge J, Blodi B, MARINA and ANCHOR Study Groups (2011) Characteristics of patients losing vision after 2 years of monthly dosing in the phase III ranibizumab clinical trials. Ophthalmol 118:523-530

12. Martin DF, Maguire MG, Ying GS, Grunwald JE, Fine SL, Jaffe GJ (2011) Ranibizumab and bevacizumab for neovascular age-related macular degeneration. CATT research group. N Engl J Med 364: 1897-908

13. Gupta OP, Shienbaum G, Patel AH, Fecarotta C, Kaiser RS, Regillo CD (2010) A treat and extend regimen using ranibizumab for neovascular age-related macular degeneration clinical and economic impact. Ophthalmol 117:2134-40
14. Mantel I, Zografos L, Ambresin A (2008) Early clinical experience with ranibizumab for occult and minimally classic neovascular membranes in age-related macular degeneration. Ophthalmol 222:321-3

15. Mitchell P, Korobelnik JF, Lanzetta P, Holz FG, Prünta C, SchmidtErfurth U, Tano Y, Wolf S (2010) Ranibizumab (Lucentis) in neovascular age-related macular degeneration: evidence from clinical trials. Br J Ophthalmol 94:2-13

16. Finger RP, Wiedemann P, Blumhagen F, Pohl K, Holz FG (2013) Treatment patterns, visual acuity and quality-of-life outcomes of the WAVE study - a noninterventional study of ranibizumab treatment for neovascular age-related macular degeneration in Germany. Acta Ophthalmol 91:540-6

17. Tschuor P, Pilly B, Venugopal D, Gale RP (2013) Optimising assessment intervals improves visual outcomes in ranibizumab-treated agerelated neovascular degeneration: using the stability phase as a benchmark. Graefes Arch Clin Exp Ophthalmol 251:2327-30

18. Gerding H (2010) Treatment of wet AMD with less than 12 injections of ranibizumab per year. Klin Monbl Augenheilkd 227:284-7

19. Ersoy L, Ristau T, Kirchhof B, Liakopoulos S (2013) Response to anti-VEGF therapy in patients with subretinal fluid and pigment epithelial detachment on spectral-domain optical Cohen tomography. Graefes Arch Clin Exp OphthalmolThis is Graefes Arch Clin Exp Ophthalmol (2014) Jun 252(6):889-97

20. Konstantinidis L, Mantel I, Zografos L, Ambresin A (2010) Intravitreal ranibizumab in the treatment of choroidal neovascularization associated with idiopathic central serous chorioretinopathy. Eur J Ophthalmol 20:955-8

21. Roisman L, Ribeiro JC, Fechine FV, Lavinsky D, Moraes N, Campos M, Farah ME (2014) Does microperimetry have a prognostic value in central serous chorioretinopathy? Retina. 34(4):713-8

22. Fassnacht-Riederle H, Becker M, Graf N, Michel S (2014) Effect of aflibercept in insufficient responders to prior anti-VEGF therapy in neovascular AMD. Graefes Arch Clin Exp Ophthalmol Graefes Arch Clin Exp Ophthalmol. doi:10.1007/s00417-014-2589-3

23. Yonekawa Y, Andreoli C, Miller JB, Loewenstein JI, Sobrin L, Eliott D, Vavvas DG, Miller JW, Kim IK (2013) Conversion to aflibercept for chronic refractory or recurrent neovascular age-related macular degeneration. Am J Ophthalmol 156:29-35

24. Cho H, Shah CP, Weber M, Heier JS (2013) Aflibercept for exudative AMD with persistent fluid on ranibizumab and/or bevacizumab. Br J Ophthalmol 97:1032-5

25. Bakall B, Folk JC, Boldt HC, Sohn EH, Stone EM, Russell SR, Mahajan VB (2013) Aflibercept therapy for exudative age-related macular degeneration resistant to bevacizumab and ranibizumab. Am J Ophthalmol 156:15-22 